\title{
A Supervisory Control Structure for Voltage-Controlled Islanded DC Microgrids
}

\author{
Alessio La Bella*, Pulkit Nahata*, Giancarlo Ferrari-Trecate
}

\begin{abstract}
In this work, we propose a supervisory control structure in islanded DC microgrids such that a well scheduled and balanced utilization of various resources is achieved. Our supervisory control layer rests on top of a voltage-controlled primary layer and comprises a secondary layer, which receives power references from an energy management system. The secondary layer translates these power into appropriate voltage references by solving an optimization problem. These references act as an input for the primary voltage controllers. We show that the unconstrained secondary optimization problem is always feasible. Moreover, since the voltages can only be enforced at the generator nodes, we provide a novel condition to guarantee the uniqueness of load voltages and power injection of the generation units. Indeed, in the absence of uniqueness, for fixed generator voltages, the load nodes and power injections may be different than planned. This can result in violation of operational limits causing damage to the connected loads. Moreover, this uniqueness condition can be verified at each load node by utilizing local load parameters, and does not require any information about microgrid topology. The functioning of the proposed architecture is tested via simulations.
\end{abstract}

\section{INTRODUCTION}

Microgrids (mGs) are electric networks comprising different devices such as distributed generation units (DGUs) interfaced with power-electronic converters, energy storage units (ESSs), and loads. MGs can operate in grid-connected and islanded modes, and are compatible with both $\mathrm{AC}$ and DC operating paradigms [1], [2], [3]. In particular, DC microgrids, due to their ability to interface naturally with renewable energy sources (for instance PV modules), batteries, and electronic loads (various appliances, LEDs, electric vehicles, etc), have gained traction in the recent years [4], [5].

The overall control of an islanded DC microgrid (DCmG) is a multi-objective problem spanning different control stages, time scales, and physical layers. For a stable and economic operation of a microgrid, a hierarchical control scheme is generally employed [4], [5], [6]. The primary control layer, acting at the component level, is responsible for voltage stability, which is crucial for islanded DCmGs. In this work, we consider the reference setting where the DGUs are equipped with decentralized primary controllers designed

\footnotetext{
* indicates equal contribution. This work has received support from the Research Fund for the Italian Electrical System in compliance with the Decree of Minister of Economic Development April 16, 2018 and the Swiss National Science Foundation under the COFLEX project (grant number 200021_169906).

A. La Bella is with Dipartimento di Elettronica, Informazione e Bioingegneria, Politecnico di Milano, Italy. Email: alessio.labella@polimi.it

P. Nahata and G. Ferrari-Trecate are with Automatic Control Laboratory, École Polytechnique Fédérale de Lausanne (EPFL), Switzerland. Email: \{pulkit.nahata, giancarlo.ferraritrecate\}@epfl.ch

Corresponding author: P. Nahata
}

to track suitable voltage references. To this purpose, several approaches, for example based on droop control [2], [4] and plug-and-play control [7], [8], have been proposed in the literature.

Primary controllers, however, are unable to account for various operational and economic constraints necessary for continuous and proper functioning of the islanded DCmG. High-level supervisory control architectures are, therefore, necessary to coordinate the voltage references provided to the primary layers. A common solution is to exploit an energy management system (EMS), which can meet specified power and energy objectives while respecting generation constraints and other economic objectives like optimal power dispatch, load sharing, and battery management. Flowchartbased EMS encompassing multiple case scenarios are discussed in [9], [10] whereas the use of optimization methods and predictive algorithms to design EMS is investigated in [11], [12].

In general, EMS, e.g. based on stochastic or mixed-integer optimization algorithms, utilize power balance equations and, in addition to their capability to consider operational constraints, provide optimal power set-points [13], [14], [15], [16]. When the primary layer is voltage controlled, the EMS power references need to be translated into suitable voltage references. Such a translation is not straightforward for $\mathrm{mGs}$ with meshed topologies and, effectively, requires the solution of power flow equations. Moreover, considering that the voltages can solely be enforced by the DGUs, a unique voltage equilibrium may fail to exist at the load buses in the presence of nonlinear loads (for example constant power loads) [17].

Leveraging the availability of grid-stabilizing primary voltage controllers, in this work we propose a supervisory control structure situated atop the primary layer and composed of a secondary and a tertiary layer. The secondary control, acting as an interface between the primary and the tertiary layer, converts the power signals provided by the tertiary level into voltage references, which are tracked by the local voltage regulators. An EMS sits at the tertiary level, and facilitates a smooth off-grid operation by providing desired power references to the secondary layer. The focus of this work is on the properties of the secondary controller and the precise design of the EMS is not considered.

Different from [9], [10], [16], we study an islanded voltaged-controlled $\mathrm{DCmG}$ with arbitrary topology and equipped with ZIP (constant impedance, constant current, and constant power) loads. In particular, we present a secondary control structure, which solves an optimization problem based on the power flow equations to generate 


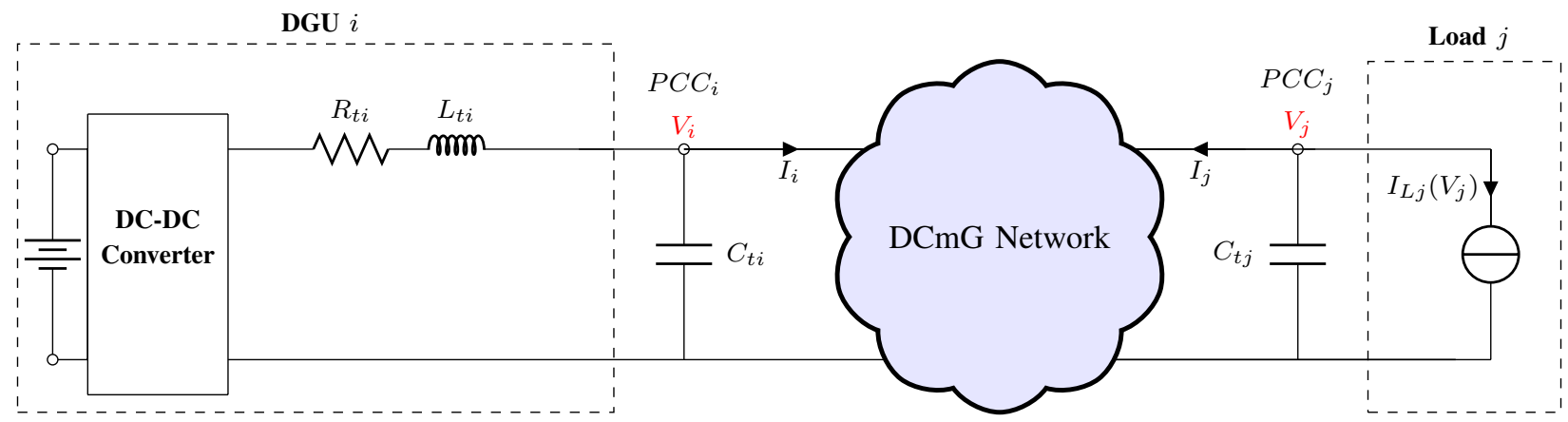

Fig. 1: Representative diagram of the DCmG network with DGUs and loads.

suitable voltage references, while taking into account the converter- and network- losses. We prove that this optimization problem is always feasible, if voltage and generation constraints are neglected. The existence of solution to the power flow equations, necessary for the feasibility of the optimization problem, has been addressed in [18], [19] with fixed DGUs voltages. Nevertheless, the provided conditions for existence can not be used directly as the DGU voltage references are free optimization variables and not known apriori. Furthermore, as a complement, we also provide a necessary condition for the solvability of the stated optimization problem.

We highlight that the voltages can only be enforced at generator nodes and therefore, the uniqueness of load voltages is necessary for attaining the predefined operational objectives. Indeed, if the voltages appearing at the load nodes are different from the ones anticipated by the secondary layer, permissible voltage limits may be violated and consequently, DGUs fail to track the optimal power set-points provided by the EMS. In this respect, we provide a novel condition for the uniqueness of load voltages and DGU power injections. The uniqueness of voltages has also been addressed in [18], where the deduced condition depends on the generator voltages and the topological parameters of the network. Here, we provide a simpler condition that depends only on local load parameters and can be easily taken into account while designing the DCmG network.

The model of DCmG along with the derivation of power and current balance equations are presented in Section II The supervisory control structure and the detailed secondary layer design is discussed in III. Simulations validating theoretical results are provided in Section IV. Finally, conclusions are drawn in Section V.

\section{A. Preliminaries and notation}

Sets, vectors, and functions: We let $\mathbb{R}$ (resp. $\mathbb{R}_{>0}$ ) denote the set of real (resp. strictly positive real) numbers. Given $x \in \mathbb{R}^{n},[x] \in \mathbb{R}^{n \times n}$ is the associated diagonal matrix with $x$ on the diagonal. The inequality $x \leq y$ for vectors $x, y \in \mathbb{R}^{n}$ is component-wise, that is, $x_{i} \leq y_{i}, \quad \forall i \in 1, \ldots, n$. For a finite set $\mathcal{V}$, let $|\mathcal{V}|$ denote its cardinality. Given a matrix $A \in$ $\mathbb{R}^{n \times m},(A)_{i}$ denotes the $i^{t h}$ row. The notation $A \succ 0, A \succeq$ $0, A>0$, and $A \geq 0$ represents a positive definite, positive semidefinite, positive, and nonnegative matrix, respectively. Throughout, $\mathbf{1}_{n}$ and $\mathbf{0}_{n}$ are the $n$-dimensional vectors of unit and zero entries, and $\mathbf{0}$ is a matrix of all zeros of appropriate dimensions. Given a weighted graph $\mathcal{G}(\mathcal{V}, \mathcal{E})$, with $\mathcal{V}$ the set of nodes and $\mathcal{E}$ the set of edges, its Laplacian matrix $L \in \mathbb{R}^{|\mathcal{V}| \times|\mathcal{V}|}$ is defined as

$$
L=A \mathbf{1}_{|\mathcal{V}|}-A,
$$

where $A$ is the adjacency matrix of $\mathcal{G}$ collecting edges weights and is defined as

$$
a_{i j}=\left\{\begin{array}{rl}
w_{i j} & \text { if }(i, j) \in \mathcal{E} \\
0 & \text { otherwise }
\end{array} .\right.
$$

\section{MOdEl OF DC MiCROGRIDS}

In this section, we start by describing the electric model of a DCmG comprising multiple DGUs connected to each other via power lines. The electric interconnections in an DCmG are modeled as an undirected connected graph $\mathcal{G}=(\mathcal{V}, \mathcal{E})$. $\mathcal{V}$ is partitioned into two sets: $\mathcal{D}=\{1, \ldots, n\}$ is the set of DGUs and $\mathcal{L}=\{n+1, \ldots, n+m\}$ is the set of loads. The edges represent the interconnecting lines of the $\mathrm{mG}$. As shown in Figure 1, each DGU and load is interfaced with the ImG through a point of common coupling (PCC). The DGUs comprise a DC voltage source, a DC-DC converter, and a series $R L C$ filter. Each of these DGUs is equipped with local voltage regulators (not shown in Figure 1), which forms the primary control layer. The main objective these controllers is to ensure that the voltage at each DGU's PCC tracks a reference voltage $V_{\text {ref, } i}$ provided by the supervisory control layer (see Section III). In steady state, the inductances and capacitances can be neglected and the current-voltage relation is given by the identity $I=B \Gamma B^{T} V=Y V$, where $B \in \mathbb{R}^{(n+m) \times|\mathcal{E}|}$ is the incidence matrix of $\mathcal{G}, I$ is the vector of PCC currents, $V$ is the vector containing PCC voltages, $\Gamma$ is the diagonal matrix of line conductances, and $Y \in \mathbb{R}^{(n+m) \times(n+m)}$ is the network admittance matrix [20]. On partitioning the nodes into DGUs and loads, the relation can be rewritten as

$$
\begin{aligned}
{\left[\begin{array}{c}
I_{G} \\
I_{L}
\end{array}\right] } & =\left[\begin{array}{ll}
B_{G} R^{-1} B_{G}^{T} & B_{G} R^{-1} B_{G}^{T} \\
B_{L} R^{-1} B_{G}^{T} & B_{L} R^{-1} B_{G}^{T}
\end{array}\right]\left[\begin{array}{c}
V_{G} \\
V_{L}
\end{array}\right], \\
& :=\left[\begin{array}{ll}
Y_{G G} & Y_{G L} \\
Y_{L G} & Y_{L L}
\end{array}\right]\left[\begin{array}{l}
V_{G} \\
V_{L}
\end{array}\right]
\end{aligned}
$$


where $V_{G}=\left[V_{1}, \ldots, V_{n}\right]^{T}, V_{L}=\left[V_{n+1}, \ldots, V_{n+m}\right]^{T}$, $I_{G}=\left[I_{1}, \ldots, I_{n}\right]^{T}$, and $I_{L}=\left[I_{n+1}, \ldots, I_{n+m}\right]^{T}$. The subscripts $G$ and $L$ indicate the DGUs and loads, respectively. Throughout this work, the following assumption is made.

Assumption 1: The PCC voltage $V_{i}$ is strictly positive for all $i \in \mathcal{V}$.

Remark 1 (Stability under primary voltage control): It is assumed that the primary controllers achieve offset-free voltage tracking and guarantee the stability of the entire DCmG network. Indeed, if the DGU voltages are not stabilized, they can increase beyond a critical level, resulting in damage to the connected loads. The reader is deferred to [5], [21], [8], [22] and the references therein for further details concerning design of stabilizing primary controllers.

Load model: Depending upon the type of load, the functional dependence on the PCC voltage changes and the term $I_{L j}\left(V_{j}\right)$ takes different expressions. Prototypical load models that are of interest include the following:

1) constant-current loads: $I_{L I j}=\bar{I}_{L j}$,

2) constant-impedance loads: $I_{L Z}\left(V_{j}\right)=Y_{L j} V_{j}$, where $Y_{L j}=1 / R_{L j}>0$ is the conductance of the $j^{t h}$ load, and

3) constant-power loads:

$$
I_{L P j}\left(V_{j}\right)=V_{j}^{-1} \bar{P}_{L j}
$$

where $\bar{P}_{L j}>0$ is the power demand of the load $j$.

To refer to the three load cases above, the abbreviations I, Z, and $\mathrm{P}$ are often used [23]. The analysis presented in this article will focus on the general case of a parallel combination of the three loads, thus on the case of ZIP loads, which are modeled as

$$
I_{L j}\left(V_{j}\right)=\bar{I}_{L j}+Y_{L j} V_{j}+V_{j}^{-1} \bar{P}_{L j} .
$$

Based on the current directions depicted in Figure 1, it is evident that $I_{L j}\left(V_{j}\right)=-I_{j}, j \in \mathcal{L}$. Using (3), one can simplify (1) as

$$
\begin{aligned}
& I_{G}=Y_{G G} V_{G}+Y_{G L} V_{L} \\
& 0=Y_{L G} V_{G}+Y_{L L} V_{L}+Y_{L} V_{L}+\bar{I}_{L}+\left[V_{L}\right]^{-1} \bar{P}_{L},
\end{aligned}
$$

where $Y_{L} \in \mathbb{R}^{m \times m}$ is the diagonal matrix of load admittances. The vectors $\bar{I}_{L}$ and $\bar{P}_{L}$ collect consumptions of I and $\mathrm{P}$ loads, respectively. Note that the power $P_{G i}$ produced by an individual DGU is the sum of power injected into the network and the filter losses. Equivalently,

$$
P_{G}=\left[V_{G}\right] I_{G}+\left[I_{G}\right] R_{G} I_{G}
$$

where $R_{G} \in \mathbb{R}^{n \times n}$ is a diagonal matrix collecting filter resistances and $I_{G}$ is the vector of DGU filter currents. On pre-multiplying (4a) with $\left[V_{G}\right]$, and by using (5), one can rewrite (4) as

$$
\begin{aligned}
f_{G}\left(V_{G}, V_{L}, P_{G}\right) & =\left[V_{G}\right] Y_{G G} V_{G}+\left[V_{G}\right] Y_{G L} V_{L} \\
& +\left[I_{G}\right] R_{G} I_{G}-P_{G}=0 \\
f_{L}\left(V_{G}, V_{L}\right)= & Y_{L G} V_{G}+Y_{L L} V_{L}+Y_{L} V_{L} \\
+ & Y_{L} V_{L}+\bar{I}_{L}+\left[V_{L}\right]^{-1} \bar{P}_{L}=0
\end{aligned}
$$

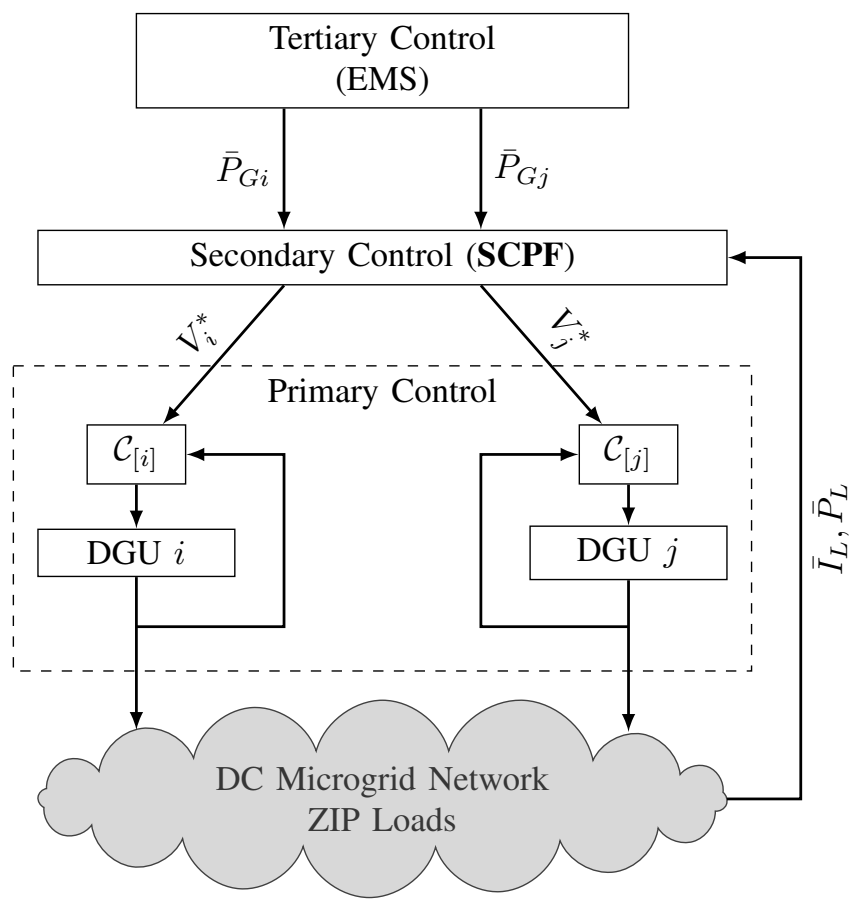

Fig. 2: Hierarchical control scheme for DC microgrids.

The equation (6) and (7) fundamentally depict the power balance and current balance at DGU and load nodes, respectively. These equations are essential for the design of secondary supervisory controller, which are discussed in the ensuing sections.

\section{SUPERVISORY CONTROL IN DC MICROGRIDS}

As shown in Figure 2, the EMS at the tertiary level sends power references to secondary layer. In this section, we discuss the detailed functioning of the secondary control layer. We would like to highlight that the secondary controller acts at a much slower time scale, compared to the primary layer. Therefore, it is assumed that the microgrid is in equilibrium with the voltage controllers tracking the reference voltage. For the secondary layer to translate power signals into appropriate voltage references, it is necessary to utilize the equilibrium relations (6), (7) linking these two variables.

\section{A. Secondary control based on power flow equations}

The secondary control is designed to track the power references provided by the EMS, denoted as $\bar{P}_{G}$. In order to achieve this goal, the secondary layer solves an optimization problem, whose objective is to minimize the difference between the reference power $\bar{P}_{G}$ and the DGU input power $P_{G}$ under the equilibrium relations (6) and (7). We first consider the following simplified version of the optimization problem, where constraints on voltages and generator power are neglected. 


\section{Secondary Power Flow (SPF):}

$$
J_{S P F}\left(\bar{P}_{G}, \bar{P}_{L}, \bar{I}_{L}\right)=\min _{V_{G}, V_{L}, P_{G}}\left\|P_{G}-\bar{P}_{G}\right\|_{2}
$$

subject to

$$
\begin{aligned}
& f_{G}\left(V_{G}, V_{L}, P_{G}\right)=0 \\
& f_{L}\left(V_{G}, V_{L}\right)=0
\end{aligned}
$$

As noticeable from Figure 2, the SPF layer requires the updated load consumption $\left(\bar{P}_{L}, \bar{I}_{L}\right)$ and the power references $\bar{P}_{G}$ in order to solve (8). We define $\mathcal{X}$ to be the set of all $\left(V_{G}, V_{L}, P_{G}\right)$ that satisfy (8b)-(8c) simultaneously. Hereafter, we will discuss necessary and sufficient conditions ensuring that the set $\mathcal{X}$ is nonempty, i.e, the solutions to nonlinear equations (8b)-(8c) exist.

Proposition 1: The feasible set $\mathcal{X}$ is non-empty . In particular, for all $\bar{P}_{L} \in \mathbb{R}^{m}$ and $\bar{I}_{L} \in \mathbb{R}^{m}$, the following statements hold:

1) The equation (8c) is always solvable.

2) The solvability of (8c) implies that (8b) is solvable.

Proof: The proof is provided in [24] and is skipped due to space constraints.

Proposition 1 guarantees the feasibility of SPF. We now discuss optimality. If SPF achieves the optimal cost $J_{S P F}^{*}=$ 0 , it implies that a voltage solution exists such that the power references $\bar{P}_{G}$ are exactly tracked by the DGUs. This condition can not be achieved for any value of $\left(\bar{P}_{L}, \bar{I}_{L}, \bar{P}_{G}\right)$. The following proposition, inspired by [25], presents a necessary condition that must hold for $J_{S P F}^{*}=0$. The proof, however, is different since here also DGU filter losses are taken into account.

Proposition 2: If the SPF achieves the optimal cost $J_{S P F}^{*}=0$, then

$$
\sum_{\forall i \in \mathcal{D}} \bar{P}_{G} \geq \sum_{\forall i \in \mathcal{L}} \bar{P}_{L}-\frac{1}{4} \bar{I}_{L}^{T} \tilde{Y}_{G G}^{-1} \bar{I}_{L}
$$

where $\tilde{Y}_{G G}=Y_{G G}-Y_{G L}^{T}\left(Y_{L L}+Y_{L}\right) Y_{G L}$.

Proof: The proof is omitted for the sake of brevity of presentation. The reader is deferred to [24] for a detailed proof.

Remark 2: It is highlighted that the necessary condition (9) depends only on the network parameters and load consumption. Therefore, it can be incorporated in the EMS optimization problem as a constraint for the choice of the power references $\bar{P}_{G}$.

In a real DCmG, the power output $P_{G}$ is constrained by physical limits of the DGUs. Moreover, the components of the DCmG are designed to operate around the nominal voltage. Hence, both nodal voltages and DGU powers must respect certain constraints, which are not incorporated in the aforementioned SPF. Consequently, we now introduce the following constrained optimization problem with additional operational constraints.

\section{Secondary Constrained Power Flow (SCPF):}

$$
\begin{aligned}
J_{S C P F}\left(\bar{P}_{G}, \bar{P}_{L}, \bar{I}_{L}\right)= & \min _{V_{G}, V_{L}, P_{G}}\left\|P_{G}-\bar{P}_{G}\right\|_{2} \\
\text { subject to } & \\
& f_{G}\left(V_{G}, V_{L}, P_{G}\right)=0 \\
& f_{L}\left(V_{G}, V_{L}\right)=0 \\
& V_{G}^{\text {min }} \leq V_{G} \leq V_{G}^{\text {max }} \\
& V_{L}^{\text {min }} \leq V_{L} \leq V_{L}^{\text {max }} \\
& P_{G}^{\text {min }} \leq P_{G} \leq P_{G}^{\text {max }}
\end{aligned}
$$

While the feasibility of SPF is always ensured by Proposition 1, this may not be true for SCPF due to the presence of additional constraints (10d)-(10f). However, if the DCmG is properly designed, a feasible solution of SCPF should always exist. In fact, the infeasibility of the SCPF implies the absence of sufficient power generation to satisfy the load demand and losses in the allowed voltage range.

Next we study the properties of an optimizer $\mathbf{x}^{*}=\left(V_{G}^{*}, V_{L}^{*}, P_{G}^{*}\right)$ of $\mathbf{S C P F}$, assuming it exists. As mentioned before, the secondary control layer acts as an interface between the EMS (tertiary layer) and the local voltage regulators (primary layer). The voltage $V_{G}^{*}$ obtained from the SCPF is transmitted as a reference to the primary voltage controllers of the DGUs. We highlight that the component $V_{G}^{*}$ of $\mathbf{x}^{*}$ can be directly imposed since the load nodes are not equipped with voltage controllers and the generators are not controlled to track power references. Therefore, it is important to guarantee that, for a given voltage reference $V_{G}^{*}, P_{G}^{*}$ is the power produced by the DGUs and $V_{L}^{*}$ appears at the load nodes. This implies that for a fixed $V_{G}^{*}$, the unique solution satisfying the power flow equation (6)-(7) must be $V_{L}=V_{L}^{*}, P_{G}=P_{G}^{*}$. We show the uniqueness by means of the following theorem.

Theorem 1: Consider the solution $\mathbf{x}^{*}=\left(V_{G}^{*}, V_{L}^{*}, P_{G}^{*}\right)$ from the SCPF optimization problem. For a fixed $V_{G}^{*}$, the pair $\left(V_{L}^{*}, P_{G}^{*}\right)$ is the unique solution of (6)-(7) in the set $\mathcal{Y}=\left\{\left(V_{L}, P_{G}\right): V_{L}>V_{L}^{\text {min }}, P_{G} \in \mathbb{R}^{n}\right\}$ if

$$
\bar{P}_{L i}<\left(V_{L i}^{m i n}\right)^{2} Y_{L i}, \quad \forall i \in \mathcal{L} .
$$

Proof: The proof is removed due to lack space and can be found in [24].

Remark 3: (Condition (11) and stability) The uniqueness condition (11) essentially limits the power consumption of $\mathrm{P}$ loads. As shown in [8], due to the negative impedance introduced by the P loads, their power consumption $P_{L, i}<$ $\left(V_{i}^{*}\right)^{2} Y_{L, i}, i \in \tilde{\mathcal{L}}$ in order to guarantee stability. Since $V_{i}^{*}$ is the solution of SCPF, $V_{i}^{*} \geq V_{i}^{m i n}$. Therefore, by satisfying (11), one can simultaneously guarantee the uniqueness of load voltages and the stability of the DCmG.

\section{NumericAl RESUlts}

In this section, we aim to show the performance of the proposed secondary control layer in a simulation framework. We consider a DCmG composed of 2 DGUs (interfaced with synchronous Buck converters) and 4 loads as shown in Figure 


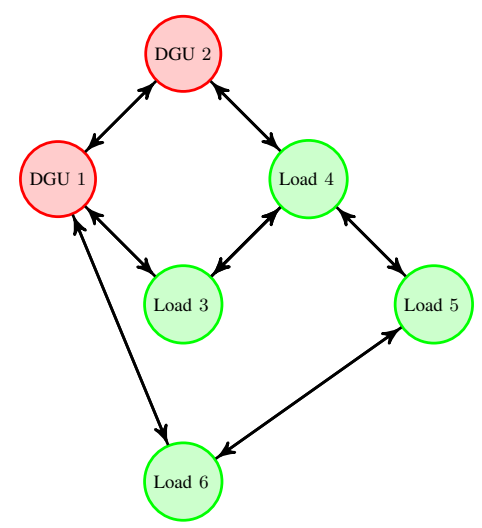

Fig. 3: Sample DcmG network composed of 2 DGUs and 4 load nodes

3. The reader is refereed to [21] for the electrical parameters of the DCmG. The DGUs are controlled by primary voltage controllers studied in [8]. The loads are standard ZIP loads. However, the power and current absorption of the loads at node 3 and node 4 are varying as depicted in Figure 4. We highlight that the loads are designed in a fashion such that (11) always holds.
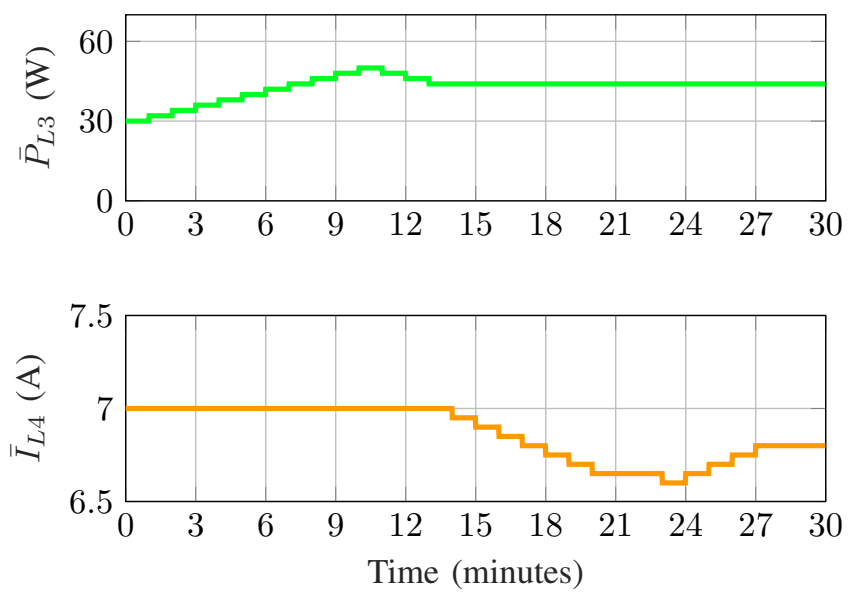

Fig. 4: Power and current variations at load 3 and 4.

The power set-points for the DGUs, denoted as $\bar{P}_{G 1}, \bar{P}_{G 2}$, are assumed to be constant for the whole simulation, even though they are normally provided by an EMS. The secondary layer runs with a sampling time of 3 minutes with the goal of tracking the received power references despite

\begin{tabular}{|c|c|}
\hline$\left(V_{G}^{\min }, V_{G}^{\max }\right)$ & $(45,55) \mathrm{V}$ \\
$\left(V_{L}^{\min }, V_{L}^{\max }\right)$ & $(45,55) \mathrm{V}$ \\
$\bar{P}_{G 1}$ & $400 \mathrm{~W}$ \\
$\bar{P}_{G 2}$ & $650 \mathrm{~W}$ \\
$\left(P_{G 1}^{\min }, P_{G 1}^{\max }\right)$ & $(0,400) \mathrm{W}$ \\
$\left(P_{G 2}^{\min }, P_{G 2}^{\max }\right)$ & $(0,700) W$ \\
\hline
\end{tabular}

TABLE I: Secondary control parameters.

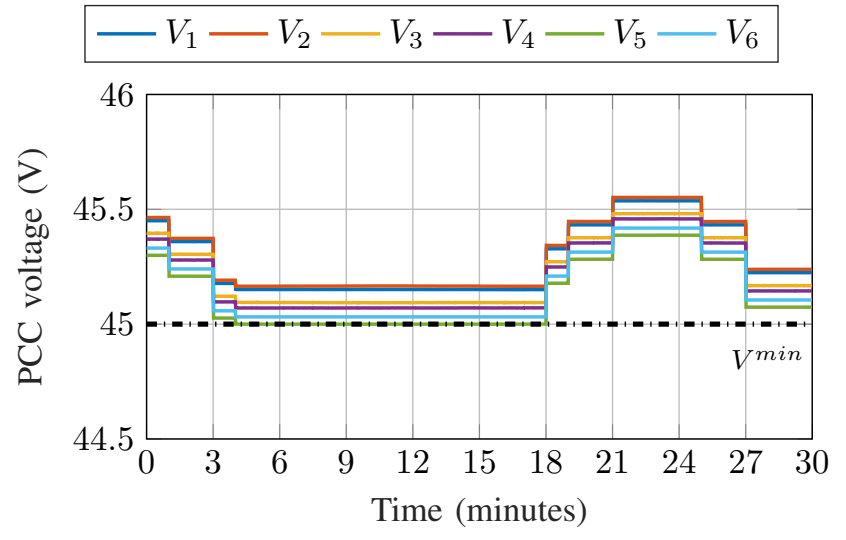

Fig. 5: Voltages in the DCmG network.
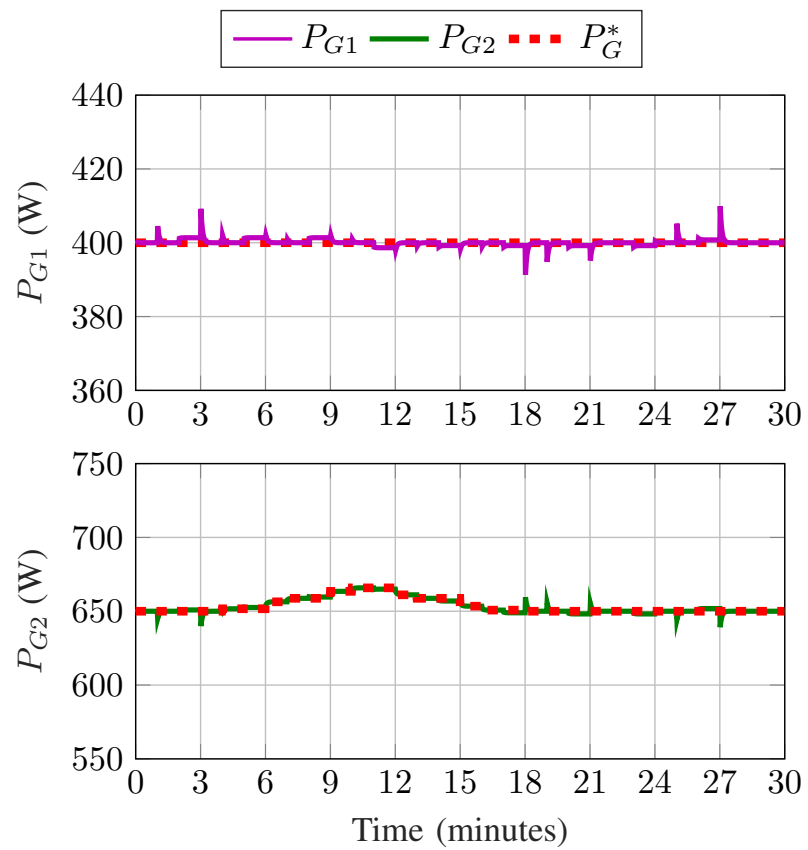

Fig. 6: Power generated by the DGUs.

the aforementioned load variations. The $\mathrm{DCmG}$ parameters considered in the secondary layer are given in Table I. As shown in Figure 5, the secondary control layer manipulates the voltage references of the DGUs at each sampling time, maintaining the voltages in the allowed range. However, in order to keep the voltages above the prescribed lower bound between $t=3 \mathrm{~min}$ and $t=18 \mathrm{~min}$, the secondary control layer does not track $\bar{P}_{G 2}$ and is forced to increase the generated power by the DGU2, since DGU1 is already at its maximum limit (see Table I). From $t=18 \mathrm{~min}$ to the end of the simulation, since the load variations do not cause any voltage issues, the EMS power references are perfectly tracked by providing the suitable voltage set-points to the primary controllers. The transients seen in Figure 6 are due to the primary voltage controllers responsible for emulating the higher-level commands at the component level. 


\section{CONCLUSIONS}

In this work, we proposed a supervisory control structure for islanded DCmGs, where a secondary layer receives power references from an EMS and translates it into voltage references for the primary layer. More specifically, the voltage references are generated by solving an optimization problem at the secondary layer, which can incorporate practical operational constraints. Furthermore, we studied the wellposedness of the optimization problem by discussing its feasibility and deduced a novel condition for the uniqueness of generator voltages and DGU power injections.

The development of a comprehensive EMS at tertiary level is deferred for future work. Further developments can also focus on solving the proposed optimization problem in a distributed and efficient manner.

\section{REFERENCES}

[1] A. La Bella, S. Negri, R. Scattolini, and E. Tironi, "A two-layer control architecture for islanded AC microgrids with storage devices," in 2018 IEEE Conference on Control Technology and Applications (CCTA). IEEE, 2018, pp. 1421-1426.

[2] Q. Shafiee, J. M. Guerrero, and J. C. Vasquez, "Distributed Secondary Control for Islanded Microgrids : A Novel Approach," IEEE Transactions on Power Electronics, vol. 29, no. 2, pp. 1018-1031, 2014.

[3] M. Babazadeh and H. Karimi, "A robust two-degree-of-freedom control strategy for an islanded microgrid," IEEE Transactions on power delivery, vol. 28, no. 3, pp. 1339-1347, 2013.

[4] T. Dragičević, X. Lu, J. C. Vasquez, and J. M. Guerrero, "DC microgrids part I: A review of control strategies and stabilization techniques," IEEE Transactions on Power Electronics, vol. 31, no. 5, pp. 4876-4891, 2016.

[5] L. Meng, Q. Shafiee, G. Ferrari-Trecate, H. Karimi, D. Fulwani, X. Lu, and J. M. Guerrero, "Review on control of DC microgrids and multiple microgrid clusters," IEEE Journal of Emerging and Selected Topics in Power Electronics, vol. 5, no. 3, pp. 928-948, 2017.

[6] A. Bidram and A. Davoudi, "Hierarchical structure of microgrids control system," IEEE Transactions on Smart Grid, vol. 3, no. 4, pp. 1963-1976, Dec 2012.

[7] A. Martinelli, P. Nahata, and G. Ferrari-Trecate, "Voltage stabilization in mvdc microgrids using passivity-based nonlinear control," in 2018 IEEE Conference on Decision and Control (CDC), Dec 2018, pp. $7022-7027$.

[8] P. Nahata, R. Soloperto, M. Tucci, A. Martinelli, and G. FerrariTrecate, "A passivity-based approach to voltage stabilization in islanded DC microgrids with ZIP loads," Tech. Rep., 2018. [Online]. Available: https://infoscience.epfl.ch/record/253266? In=en

[9] M. Kumar, S. C. Srivastava, and S. N. Singh, "Control strategies of a dc microgrid for grid connected and islanded operations," IEEE Transactions on Smart Grid, vol. 6, no. 4, pp. 1588-1601, July 2015.

[10] T. Dragičević, J. M. Guerrero, J. C. Vasquez, and D. krlec, "Supervisory control of an adaptive-droop regulated dc microgrid with battery management capability," IEEE Transactions on Power Electronics, vol. 29, no. 2, pp. 695-706, Feb 2014.

[11] A. La Bella, S. R. Cominesi, C. Sandroni, and R. Scattolini, "Hierarchical predictive control of microgrids in islanded operation," IEEE Transactions on Automation Science and Engineering, vol. 14, no. 2, pp. 536-546, 2017.

[12] M. Marzband, F. Azarinejadian, M. Savaghebi, and J. M. Guerrero, "An optimal energy management system for islanded microgrids based on multiperiod artificial bee colony combined with markov chain," IEEE Systems Journal, vol. 11, no. 3, pp. 1712-1722, 2017.

[13] C. A. Hans, P. Sopasakis, A. Bemporad, J. Raisch, and C. ReinckeCollon, "Scenario-based model predictive operation control of islanded microgrids," in 2015 54th IEEE Conference on Decision and Control (CDC), Dec 2015, pp. 3272-3277.

[14] A. Parisio, E. Rikos, and L. Glielmo, "Stochastic model predictive control for economic/environmental operation management of microgrids: An experimental case study," Journal of Process Control, vol. 43, pp. 24-37, 2016.
[15] S. R. Cominesi, A. La Bella, M. Farina, and R. Scattolini, "A multi-layer control scheme for microgrid energy management," IFACPapersOnLine, vol. 49, no. 27, pp. 256-261, 2016.

[16] A. Iovine, T. Rigaut, G. Damm, E. De Santis, and M. D. Di Benedetto, "Power management for a dc microgrid integrating renewables and storages," Control Engineering Practice, vol. 85, pp. 59-79, 2019.

[17] A. S. Matveev, J. E. Machado, R. Ortega, J. Schiffer, and A. Pyrkin, "On the existence and long-term stability of voltage equilibria in power systems with constant power loads," arXiv preprint arXiv:1809.08127, 2018.

[18] J. W. Simpson-Porco, F. Dörfler, and F. Bullo, "Voltage collapse in complex power grids," Nature communications, vol. 7, p. 10790, 2016.

[19] S. Taheri and V. Kekatos, "Power flow solvers for direct current networks," arXiv preprint arXiv:1807.03936, 2018.

[20] F. Dörfler, J. W. Simpson-Porco, and F. Bullo, "Electrical networks and algebraic graph theory: Models, properties, and applications," Proceedings of the IEEE, vol. 106, no. 5, pp. 977-1005, 2018.

[21] M. Tucci, S. Riverso, and G. Ferrari-Trecate, "Line-independent plugand-play controllers for voltage stabilization in DC microgrids," IEEE Transactions on Control Systems Technology, 2018. To appear.

[22] M. Tucci, S. Riverso, J. C. Vasquez, J. M. Guerrero, and G. FerrariTrecate, "A decentralized scalable approach to voltage control of DC islanded microgrids," IEEE Transactions on Control Systems Technology, vol. 24, no. 6, pp. 1965-1979, 2016.

[23] P. Kundur, Power System Stability and Control. McGraw-Hill, 1994.

[24] P. Nahata, A. La Bella, R. Scattolini, and G. Ferrari-Trecate, "Hierarchical control in islanded DC microgrids with flexible structures," Tech. Rep., 2019.

[25] S. Sanchez, R. Ortega, R. Grino, G. Bergna, and M. Molinas, "Conditions for existence of equilibria of systems with constant power loads," IEEE Transactions on Circuits and Systems I: Regular Papers, vol. 61, no. 7, pp. 2204-2211, 2014. 